\title{
Thermal Model of the Electro-Spark Nanomachining Process
}

\author{
Gh. Tahmasebi Pour, Y. Tahmasebi Pour, and M. Ghoreishi
}

\begin{abstract}
Electrical Discharge/Spark Machining process is one of the advanced machining processes that can machine the various complex shapes from all conductor and semiconductor materials. Wide and diverse applications of spark micromachining process in microfabrication and micro to nano miniaturization tendency is promising application of spark nanomachining process in nanofabrication. Spark nanomachining process is a thermal machining process in which thermal energy produced by electric sparks is used for machining. Therefore, thermal modeling of the spark nanomachining process is very important for accurate understanding of the process and its better application in nanotechnology. In this study, thermal model of workpiece in the spark nanomachining process has been developed. The impact of variables of the spark nanomachining process on temperature distribution in the workpiece and dimensions of the machined nano-craters were analyzed.
\end{abstract}

Index Terms-Electrical discharge / spark machining, spark nanomachining, heat transfer, thermal modeling

\section{INTRODUCTION}

Electrical Discharge/Spark Machining process is one of the modern machining processes whereby different workpieces with various complicated geometric forms can be produced from a wide range of soft and hard as well as conductive and semi-conductive materials. In the electrical discharge machining process, resulting thermal energy from electrical spark is used to remove material from the workpiece surface. The amount of material removed from the workpiece surface depends on the consumed energy level in the spark. Therefore, decreasing consumed energy by each spark may lead to reduction of dimensions of the machined craters. Furthermore, the absence of any contiguity between tool and workpiece as well as lack of any mechanical force on tool and workpiece enables the application of very tiny tools to machine very small workpieces [1].

Electrical discharge machining process, due to the unique benefits and inherent potentials, has found very successful applications in microfabrication. Rapid development of nanotechnology and need to using new methods, has led to a lot of attraction for application of the spark nanomachining process in nanofabrication.

As mentioned before, electrical discharge machining

Manuscript received July 5, 2013; revised August 28, 2013.

Gh. Tahmasebi Pour is with the Faculty of New Sciences and Technologies, University of Tehran, Tehran, Iran (e-mail: tahmasebipour@ ut.ac.ir).

Y. Tahmasebi Pour and M. Ghoreishi are with the Faculty of Mechanical Engineering, K. N. Toosi University of Technology, Tehran, Iran (e-mail: lm-tahmasebipour@araku.ac.ir, e-mail: ghoreishi@kntu.ac.ir). process is one of the thermal machining processes wherein thermal energy resulted from the electrical spark is used for material removal from the surface of the workpiece. Thus, thermal modeling of the spark nanomachining process can make more precise understanding of this process and lead to its better application in nanotechnology.

Several thermal models have been presented for electrical discharge machining process at macro and micro scale so far. Snoeys presented a thermal model for the electrical discharge machining process at macro scale [2]. In this model, a circular heat source and a semi-limited cylindrical space were assumed for heat transfer. The consumption energy per spark was assumed to be equally divided between the electrodes. Patel [3] and Dibitonto [4], with the assumption of a point heat source for the cathode and a circular heat source for the anode, reported another thermal model for macro-scale electrical discharge machining process. These models are appropriate for the electrical discharge machining process with high pulse duration and high electric current. In addition, two other models have been proposed by Singh [5] and Leonid [6] for thermal modeling of the electrical discharge machining process at macro scale.

An analytical model based on electro-thermal theory was developed for electrical discharge machining process at micro scale by Yeo [7]. In this model, a uniform circular heat source was used to modeling distribution of the heat resulting from a single electric spark on the workpiece surface. Allen simulated the micro electrical discharge machining process by using numerical analysis method [8]. In this simulation, a Gaussian circular heat source was assumed and the temperature distribution on the surface of a molybdenum workpiece was modeled.

To author's knowledge, thermal model of the spark nanomachining process has not been reported so far. In this paper, distribution of the temperature resulting from the spark nanomachining process in the surface and depth of a gold nanofilm (as a workpiece) has been modeled based on the finite-volume method.

\section{Thermal Model of Spark NanOMachining Process}

As shown in (1), a 2D differential equation of heat transfer (along the radius and depth) in a cylindrical coordinate system was used to thermal model of the workpiece in the spark nanomachining process. Plasma channel of the spark nanomachining process acts as a heat source on the workpiece surface. Thus, a heat source with a radius equal to the radius of plasma channel has been assumed on the workpiece surface. 


$$
\frac{\partial^{2} T}{\partial r^{2}}+\frac{1}{r} \frac{\partial T}{\partial r}+\frac{\partial^{2} T}{\partial Z^{2}}=\frac{1}{\alpha} \frac{\partial T}{\partial t} \quad, \quad \alpha=\frac{K}{\rho C_{P}}
$$

where, $k$ is the thermal conductivity, $\alpha$ is the heat penetration coefficient, $\rho$ is the average density of workpiece and $C p$ is the average specific heat capacity.

\section{A. Assumptions}

The following assumptions have been considered in thermal modeling of the spark nanomachining process:

1) Heat source produced by the electric spark has been considered as a circle with time-dependent radius (equal to radius of the plasma channel).

2) Heat flux of the heat source has been considered uniformly because of the nano-second spark pulse duration and nano-meter radius of the heat source in spark nanomachining process.

3) Conduction is the sole way to heat transfer across the workpiece.

4) The part of workpiece which has reached the melting point (and higher) has been assumed as the removed material.

5) Consumed energy by a single spark and its effects has been studied.

6) The average of physical properties of the material has been used.

7) The difference between density of solid and liquid states has been underestimated.

8) It is supposed that a constant percentage of spark energy is taken in by the workpiece.

9) The workpiece material is assumed isotropic.

It is assumed that the workpiece is a gold nanofilm which is prepared by sputter coating on a silicon substrate. Table I indicates gold and silicon properties.

\begin{tabular}{lcc}
\multicolumn{3}{c}{ TABLE I: GOLD AND SILICON PROPERTIES. } \\
\hline Material & $\mathrm{Au}$ & $\mathrm{Si}$ \\
\hline Density $(\mathrm{kg} / \mathrm{m} 3)$ & 19320 & 2330 \\
Thermal conductivity coefficient (w/m.k) & 297 & 83 \\
Melting heat (kj/kg) & 67 & 1658 \\
Specific heat capacity (j/kg.k) & 129 & 750 \\
& 1338 & 1696 \\
\hline
\end{tabular}

\section{B. Boundary Conditions}

Fig. 1 shows schematic form of nano-crater resulted from conduction of the spark nanomachining process on a gold nano-film, heat flux due to electrical spark and hypothetical boundary of the problem. Plasma channel of the spark nanomachining process is the source of heat generation and transmission to the workpiece surface. Hence, the radius of existing heat source on the nano-film surface has been considered equal to the radius of the plasma channel. Considering nanometric scale of the plasma channel radius and nanosecond scale of spark pulse duration in the spark nanomachining process [9], heat flux distribution has been assumed uniform.

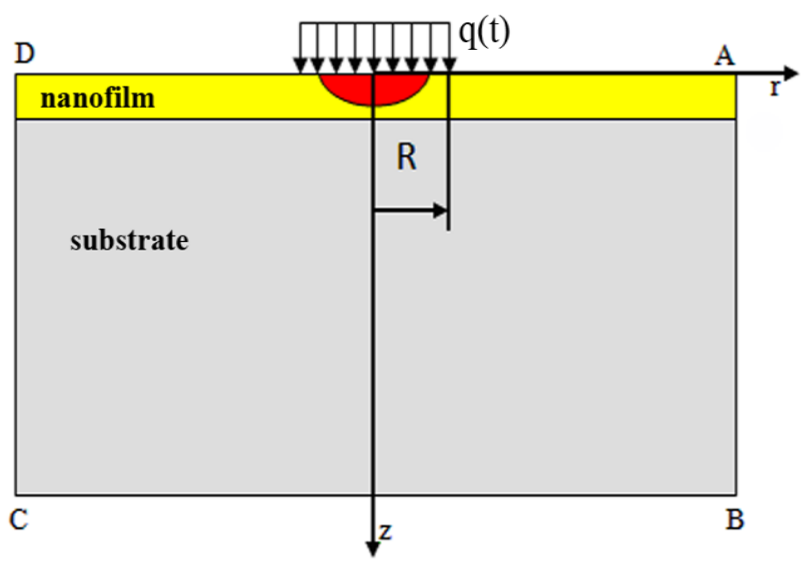

Fig. 1. Schematic diagram of the spark nanomachining process.

Temperature in $\mathrm{AB}, \mathrm{BC}$ and $\mathrm{CD}$ boundaries are taken equal to the environment temperature $(300 \mathrm{k})$. Conditions of DA boundary during pulse on-time are as follows:

$$
z=0, k \frac{\partial T}{\partial z}=\left\{\begin{array}{cc}
q(t) & 0 \prec r \prec R \\
0 & R \prec r \prec 2.5 R
\end{array}\right.
$$

In $r>2.5 R$ range, temperature has been considered constant and equal to environment temperature due to its long distant from the heat source and also its contact with a large volume of liquid dielectric. $q(t)$ is heat flux distribution function which is calculated through (3).

$$
q(t)=\frac{\mathrm{F} V I}{\pi R^{2}(t)}
$$

where, $F$ is the percentage ( $18 \%$ ) of the total power which is transmitted into the workpiece [4], $V$ is the average of spark voltage (v), $I$ is the average electric current of spark (A), $R(t)$ is radius of heat source located on the workpiece surface (equal to the plasma channel radius) (m). Plasma channel radius of the Spark nanomachining process depends on time and is computed via (4) [9].

$$
R(t)=0.8935 t^{0.8485}
$$

In this study, through solving the differential equation (1) using Finite Volume Method (FVM) by the Fluent 6.3 software, temperature distribution across the workpiece of spark nanomachining process has been modeled. For this purpose, workpieces are meshed with $10 \mathrm{~nm} \times 10 \mathrm{~nm}$ elements. Conditions of the Spark nanomachining process experiments shown in Table II, which have been tested by [9], were used to investigate impact of the process variables on temperature distribution in both surface and depth of the workpiece.

\section{RESULTS AND DISCUSSION}

Conditions of the spark nanomachining process that were practically used by [9] are shown in Table II. These conditions are used as inputs of the spark nanomachining thermal model. 
TABLE II: SPARK NANOMACHINING PROCESS CONDITIONS

\begin{tabular}{|c|c|c|}
\hline $\begin{array}{c}\text { Expt. } \\
\text { No. }\end{array}$ & $\begin{array}{c}\text { Average Consumption Power } \\
(\mathrm{w})\end{array}$ & $\begin{array}{c}\text { Pulse Duration } \\
(\mathrm{ns})\end{array}$ \\
\hline 1 & 0.15 & 4 \\
\hline 2 & 0.5 & 12 \\
\hline 3 & 0.7 & 40 \\
\hline
\end{tabular}

Temperature distribution caused by the spark nanomachining process in both surface and depth of the workpiece was modeled for various values of the process parameters. Fig. 2 shows results of the thermal modeling of workpiece during the spark nanomachining process for the condition mentioned in Table II. In this figure, temperature distribution of the workpiece $\left(\mathrm{a}_{1}, \mathrm{a}_{2}\right.$, and $\left.\mathrm{a}_{3}\right)$, temperature changes along the radius $\left(b_{1}, b_{2}\right.$, and $\left.b_{3}\right)$ and depth of the workpiece $\left(c_{1}, c_{2}\right.$, and $\left.c_{3}\right)$ have been shown for experiments 1 to 3, respectively. According to the Fig. 2, it is observed that more distance from the center of workpiece surface along the radius and depth results decrease in temperature.

Regarding Figs. 2- $a_{1}, 2-b_{1}$ and 2- $c_{1}$ and considering the melting point of $\mathrm{Au}$ and $\mathrm{Si}$, it is observed that the gold nanofilm is melted up to about $50-60 \mathrm{~nm}$ radius and $40-50 \mathrm{~nm}$ depth.

Figs. 2-a $2,2-b_{2}$, and $2-c_{2}$ illustrate the results of thermal modeling for experiment 2. According to these figures, surface of the workpiece is melted up to about $170-190 \mathrm{~nm}$ radius. Gold nanofilm is completely melted along the depth. Additionally, about $10-20 \mathrm{~nm}$ of the silicon substrate is melted, as well.

Results of the thermal modeling for experiment 3 are shown in Figs. 2-a $3,2-b_{3}$ and 2-c $c_{3}$. It is observed that the workpiece surface is melted up to radius of about 500-520 $\mathrm{nm}$ and besides the gold nanofilm, silicon substrate is melted up to about $100-110 \mathrm{~nm}$ in depth.
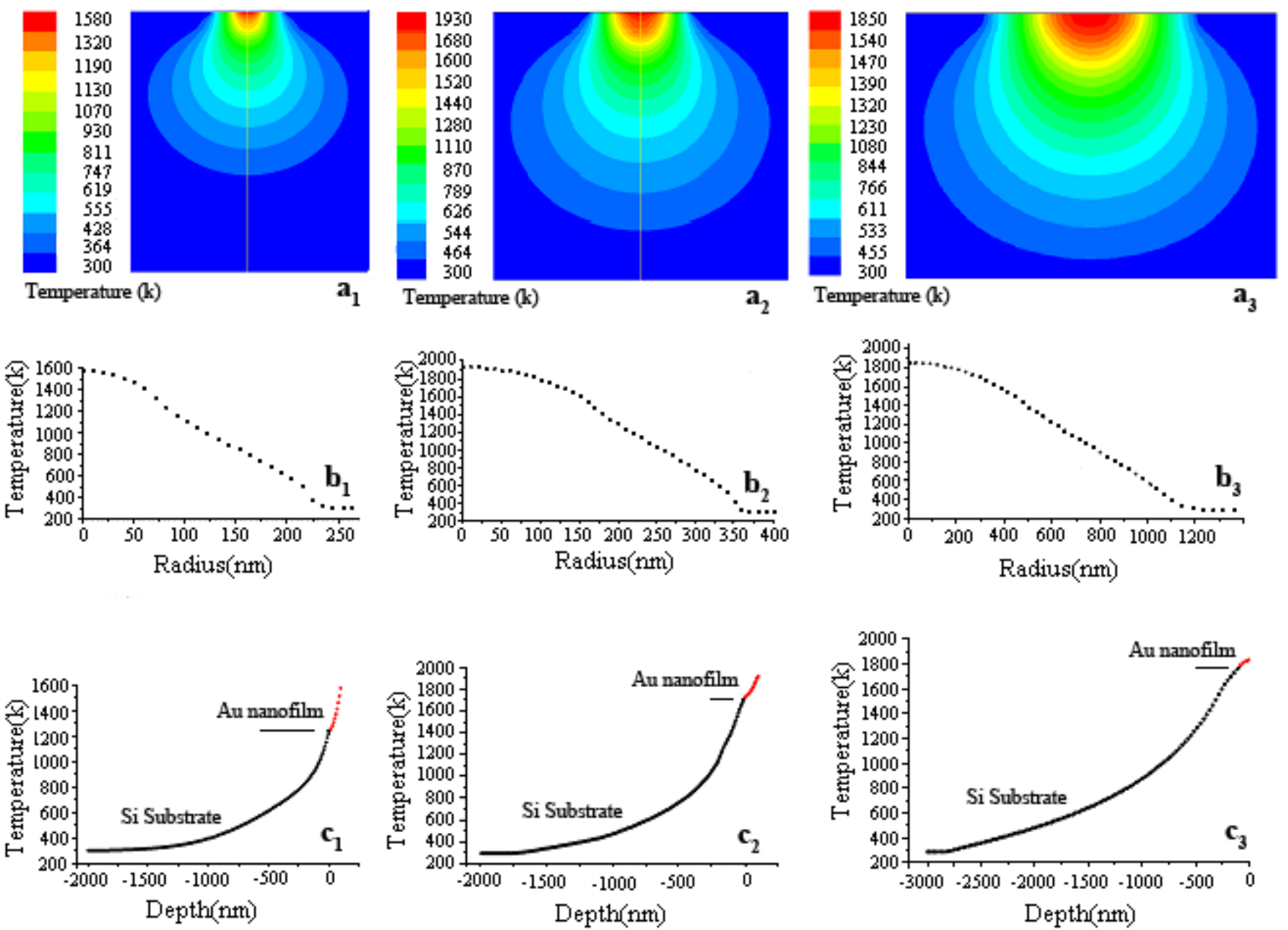

Fig. 2. Results of the model for a gold nanofilm with $100 \mathrm{~nm}$ thickness and a silicon wafer as substrate: graphical form of the temperature distribution in the workpiece $\left(a_{1}, a_{2}\right.$ and $\left.a_{3}\right)$, diagram of the temperature variations along the radius $\left(b_{1}, b_{2}\right.$ and $\left.b_{3}\right)$ and diagram of the temperature variations along depth of the workpiece and substrate $\left(c_{1}, c_{2}\right.$ and $\left.c_{3}\right)$ for the experiments 1,2 and 3 , respectively.

It can be concluded that temperature of the surface and depth of workpiece in the spark location is dependent on the consumed power and spark pulse duration. So, the higher spark power results in higher temperature of the surface and depth of the workpiece. On the other hand with the shorter spark pulse duration, more opportunity will be provided for heat transfer and the final temperature of the workpiece will decreases. Therefore, the interaction between two mentioned factors determines the temperature distribution across the surface and depth of the workpiece.

\section{CONCLUSION}

In this paper, finite volume based CFD method was used to offer a thermal model for the workpiece in the spark nanomachining process. Temperature distribution across 
both surface and depth of the workpiece for different values of the process parameters was modeled. Following results were obtained in this research:

1) Higher temperature is generated on the surface and in depth of the workpiece when the more power is consumed by the spark. On the other hand, at longer spark pulse duration, the more time is provided for heat transfer, so the final temperature of the workpiece is decreased. Therefore, interaction between two mentioned factors determines the temperature distribution across the surface and depth of the workpiece.

2) Increase in spark pulse duration increases radius of the heat source on the workpiece surface. Hence, more area of the workpiece surface is affected by the heat and more volume of the material reaches to the melting point.

\section{ACKNOWLEDGMENT}

The authors thank the financial support of University of Tehran for this research.

\section{REFERENCES}

[1] Z. Katz and C. J. Tibbles, "Analysis of micro-scale EDM process," Int. J. Adv. Manuf. Technol., vol. 25, pp. 923-928, 2005.

[2] R. Snoeys and F. S. V. Dijck, "Investigation of electro discharge machining operations by means of thermo-mathematical model," CIRP Ann., vol. 20, pp. 35-37, 1971.

[3] M. R. Patel, M. A. Barrufet, P. T. Eubank, and D. D. DiBitonto, "Theoretical models of the electrical discharge machining process: II. The anode erosion model," J. Appl. Phys., vol. 66, pp. 4104-4111, 1989.

[4] D. D. Dibitonto, P. T. Eubank, M. R. Patel, and M. A. Barrufet, "Theoretical models of the electrical discharge machining process. I. A simple cathode erosion model," J. Appl. Phys. Vol. 66, pp. 4095-40104, 1989.

[5] A. Singh and A. Ghosh, "A thermo-electric model of material removal during electric discharge machining," Int. J. Mach. Tools Manuf., vol. 39, pp. 669-682, 1999.
[6] I. S. Leonid, A. Marotta, and M. E. Alexi, "Model of workpiece erosion for electrical discharge machining process," Appl. Surf. Sci., vol. 253, pp. 797-804, 2006.

[7] S. H. Yeo, W. Kurnia et al., "Tan Electro-thermal modeling of anode and cathode in micro-EDM," Phys. D: Appl. Phys., vol. 40, pp. 2513-2521, 2007.

[8] P. Allen and X. Chen, "Process simulation of micro electro-discharge machining on molybdenum," J. Mater. Process. Technol., vol. 186, pp. 346-355, 2007.

[9] G. Tahmasebipour, "Performance and modeling of nano electrical spark machining Process," Ph.D. dissertation, Dept. Mech. Eng., Tarbiat Modares Univ., Tehran, Iran, 2009.

Gh. Tahmasebi Pour was born in Iran in 1974. He received the bachelor of science degree in mechanical engineering from the University of Tabriz, Tabriz, Iran, 1998, a master of science degree in mechanical engineering from the Amirkabir University of Technology, Tehran, Iran, 2001, and a $\mathrm{Ph} . \mathrm{D}$. degree in mechanical engineering from the Tarbiat Modares University, Tehran, Iran, 2009.

$\mathrm{He}$ is an assistant professor in the Faculty of New Sciences and Technologies at the University of Tehran in Tehran, Iran. His research interests include nano science and technology, micro/nano-scale manufacturing technologies, MEMS/NEMS, nano-mechanics, synthesis and application of nano-materials, industrial nanostructures, composites and nanocomposites, multi-scale modeling and simulation, new manufacturing science and technologies, industrial automation, and mechatronics.

Dr. Tahmasebi Pour is a recipient of the TWAS prize to young scientists in 2011, 24th Khwarizmi International Award from Iranian Research Organization for Science and Technology (IROST) in 2011, certificate and gold medal from Economic Cooperation Organization (ECO) cultural institute in 2011. He ranked 2nd in the Tarbiat Modares University entrance exam for the Ph.D. degree in Iran, 2003, and ranked 3rd in the nationwide university entrance exam for the M.Sc. degree in Iran, 1998.

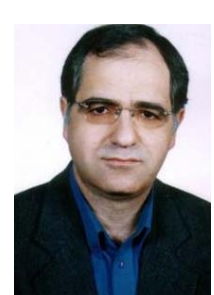

Majid Ghoreishi received the B.Sc. and M.Sc. degrees in mechanical engineering from the Sharif University of Technology, Tehran, Iran and Ph.D. degree in the area of nontraditional machining in 2001 from University of Manchester Institute of Science and Technology (UMIST), Manchester, England. His research interest are non-traditional machining, laser machining and welding electro; discharge machining (EDM); black box modeling; statistical modeling and analysis; neural networks; process optimization. 